\title{
Optimal MPR Set Selection Algorithm Based on OLSR Routing Protocol
}

\author{
Dong Siyu ${ }^{1, a}$, Zhang Hong ${ }^{2, b}$ \\ ${ }^{1}$ Department of Information Engineering, Officers College of PAP, Chengdu, Sichuan 610213, \\ China \\ ${ }^{2}$ College of Information Science and Technology, Chengdu University, Chengdu, Sichuan 610106, \\ China \\ adongsiyu@yeah.net , bcdutzh101@163.com
}

Keywords: OLSR; MPR Set; Ad Hoc; Link State; Connection Degree

\begin{abstract}
The optimal MPR (Multi Point Relay) set selection algorithm is proposed based on the researches of the OLSR (Optimized Link State Routing) protocol of the Ad Hoc network. This paper analyzes the MPR set selection problem from the point of view of mathematical collection. By abstracting one-hop neighbor nodes and the neighbors which are connected by the nodes into the set containing the subsets, we calculate the independent subset of the remaining set to generate MPR relay nodes, and thus find the MPR set with the minimum number. The simulation results show that the algorithm improves the success rate of the data transmission and enhances the network throughput.
\end{abstract}

\section{Introduction}

The mobile Ad Hoc network [1] (Mobile Ad Hoc Networks, MANET) is a kind of peer-to-peer network which the nodes have no distinction between them and also have the function of the host and the router. Ad Hoc network is a kind of multiple hops, no center, self-organized wireless network and also known as multiple hops network (Multi-hop Network) or self-organizing network (Self - organizing Network). The network has no fixed infrastructure, each node is mobile, and can be in any way to keep in touch with other nodes dynamically.

OLSR [2] protocol is an important part of the MANET routing protocol, its core technology is MPR set. This genetic algorithm is derived into four series of algorithm by using different genetic strategy and be simulated with the randomly generated topology [3]. However, the algorithm is more complicated and more difficult to achieve. Literature [4] introduced ant colony algorithm to solve minimum MPR sets which gave the improved ant colony algorithm CSACO based candidate solution. Due to ant colony algorithm using the positive feedback characteristics of information, easy to make the results into a local optimal solution, rather than the global optimal solution. This paper analyzed the cause of the problem, and proposed the corresponding improvement method to find the minimum MPR set, and finally verified the effectiveness of the improved scheme by simulation.

\section{The Characteristics Of OLSR}

OLSR is a proactive link-state routing protocol [5], mainly used in MANET network [6], and optimized the traditional LS (Link State) protocol on the basis of requirements of MANET. The OLSR's key technology is MPR (Multi-point Relay), the main optimized designs for traditional LS protocol are:

(1) Only choose some nodes as MPRS to transport controlled packets, so as to reduce its flooding scope: the chosen nodes named MPR transport packets, the other nodes only to receive the packets, don't transport them.

(2) Reduce the size of controlled packets: the nodes don't transport the all neighbor's link state message, only transport the MPR's link state, so as to reduce the size of controlled packets.

The key technology of OLSR is Multi-Point Relays (MPRs), by reducing the number of repeat forwards packets in the same area to reduce the number of control packets. 


\section{The Limitations Of Traditional OLSR}

The Meaning Of Traditional Of MPR. The traditional OLSR routing protocol uses two methods to reduce the routing cost because of link state information flood, one is using Multi-Point Relays, each node select the other nodes as part of their own one hop MPR, forwarding the link state information from the MPR, but not all hop nodes for routing through the MPR selective flooding control messages; The other is compressing the link state information [7] which described the link between MPR select node and MPR, rather than all one hop neighbor nodes link. While in the choice of MPR, classical OLSR routing protocol chooses the connection degree as reference standard algorithms.

The Problem Of Traditional MPR Choice. Because traditional OLSR routing protocols only consider the least number of hops. However, the routing protocol is only best-effort to transmitting data, without considering the actual state of congestion in the network intermediate nodes and wireless links, so it's choice of MPR set is not necessarily minimal. Therefore, the traditional OLSR routing protocol has been unable to meet the higher quality and greater variety of business requirements of mobile Ad Hoc network. So this paper improved the algorithm of the traditional OLSR protocol routing by selecting least MPR.

\section{The Improvement Of MPR Nodes}

The Problem Of Traditional MPR Nodes. The chosen process of MPR nodes, such as figure.1

(1) Choose the only two-hop neighbors and delete the all one-hop neighbor's two-hop neighbors. Then, the MPR set is $\{e, f\}$.

(2) Select the rest of the two-hop neighbors set depend on maximum connection degree until the set is empty, so the MPR set is $\{e, f, b, c, a\}$. For example, from the figure.1, the optimal number of MPR is four and the MPR set is $\{f, e, a, c\}$, but the traditional MPR set is $\{f, e, a, b, c\}$. So getting the optimal number of MPR is the key of this paper.

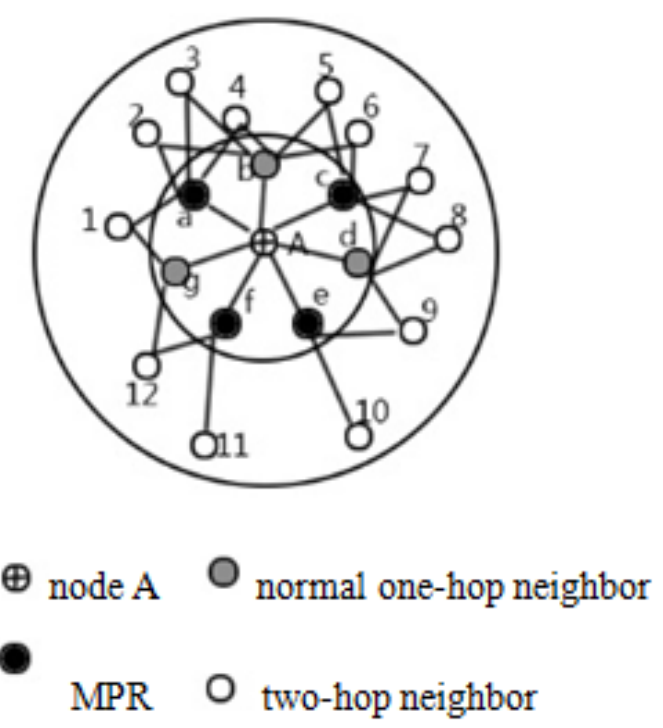

Fig.1 traditional MPR selection algorithm

The Improved Method For MRP Selection. The improved method for selection based on collection problem. From figure.2, this paper divides into three kinds of sets: 1, the two-hop neighbors set of node A: SEC $\{1,2,3,4,5,6,7,8,9\}$; 2,the one-hop neighbors set: FIR $\{a, b, c, d, e\}$; 3 , for each one-hop neighbor's set, such as node a, a $\{7,8,9\}$ for figure.3. 


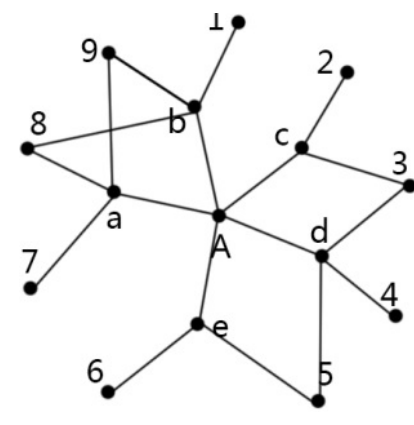

Fig.2 the net example

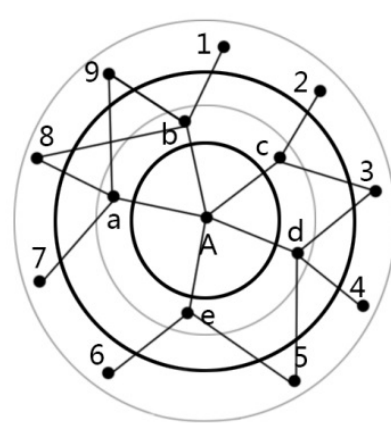

Fig.3 collection division

The key method is to find the only two-hop neighbor so as to select the MPR set which can guarantee the least number of node. The method that has the different from traditional selection which based on the maximum degree of connection depend on find the only way to carry on until the two-hop neighbor SEC $\{1,2,3, \ldots \mathrm{n}\}$ is empty.

After the first selection of MPR set, the left two-hop neighbor nodes which are not the only one-hop neighbor nodes are empty. Such as figure.4.

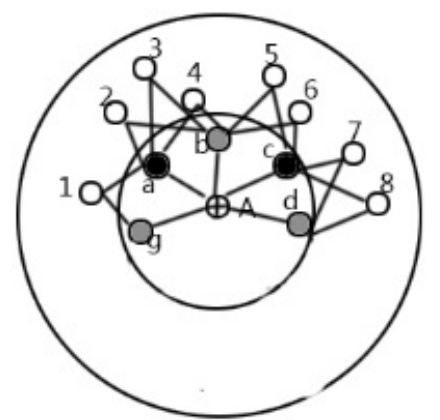

Fig.4 the first result of MPR

We can get the relation perspective abstract from figure 4 to figure 5 . The sets include $\mathrm{g}\{1\}$, $a\{1,2,3,4\}, b\{2,3,4,5,6\}, c\{5,6,7,8\}, d\{7,8\}$, the two-hop neighbors set SEC $\{1,2,3,4,5,6,7,8\}$ and the one-hop neighbors set FIR $\{\mathrm{g}, \mathrm{a}, \mathrm{b}, \mathrm{c}, \mathrm{d}\}$. There are some redundancy in all sets, so we can delete the redundancy, eg. The 'a' set includes ' $g$ ' set, so deletes ' $g$ ', but retains it's subset. When delete all the redundancy, we can get the example for figure 6 . And the question has been solved, figure 6 becomes the basic condition, we can find the program which is the only two-hop neighbor to solve it. At the same time, there has a important conclusion: through deleting the redundancy, we get the only two-hop neighbor unnecessarily, but the only two-hop neighbor must be in the redundancy.

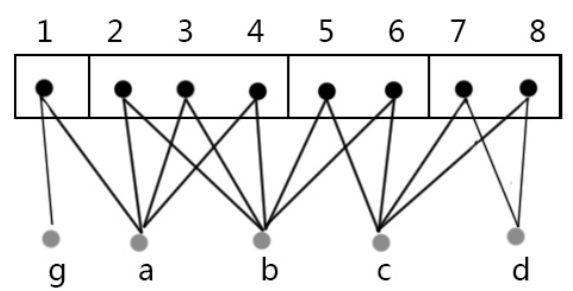

Fig. 5 the relationship of set

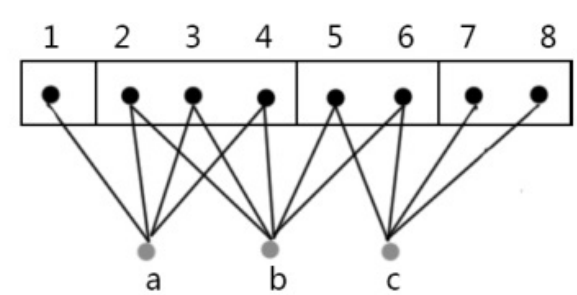

Fig.6 delete the redundancy

\section{Algorithm Description}

Step 1. Check the SEC $\{1,2,3, \ldots \mathrm{n}\}$ is empty or not;

Step 2. Find the only two-hop neighbor which the node A must reach;

Step 3. Set all the element weights of SEC to ' 0 ' and read the all element from all subset and add weights with ' 1 ', when read it over, check all the element which have weights with ' 1 ' and delete them from the subset FIR $\{a, b, c . .$.$\} , and reset the weights to ' 0$ ';

Step 4 . When find the only node which involves the signal (ring net), set the initial value to ' 0 ', and when the state changes, set the weights to ' 1 ', don't reset it;

Step 5. Use the signal's variety to judge it whether ring net or not, such as figure 7 . All the 
element's weights from SEC $\{1,2,3, \ldots\}$ are more than ' 1 ', we can delete the redundancy;

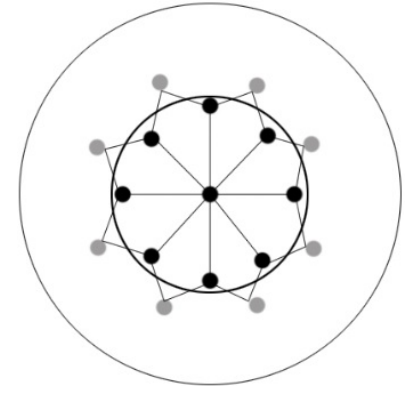

Fig.7 ring net

Step 6. When the net is ring, all the element's weights from SEC $\{1,2,3, \ldots\}$ are more than ' 1 ';

Step 7. Deleting the redundancy sets is to prevent the ring net: all the element's weights from SEC $\{1,2,3, \ldots\}$ are more than ' 2 ';

Step 8. When all the element's weights from SEC $\{1,2,3, \ldots\}$ are ' 2 ', we must destroy the net so as to guarantee the algorithm is right. Destroying the net is only to delete the random element from FIR $\{a, b, c, .$.$\} , not delete all the sub-element.$

Definition: destroying the ring net, we can get the least number of MPR: the ring net which has the even nodes, regardless of which one destroy, resulting MPR nodes $=\mathrm{N} / 2$ ( $\mathrm{N}$ is the number of hop); odd Ring: MPR nodes = $(\mathrm{N}+1) / 2$.

Proof: deleting redundant collection was bound for a minimum of MPR nodes: if a collection contains a subset which must be deleted, because if we can select a subset to the MPR, the larger set will be selected as MPR is the best selection, thus ensuring a minimal number MPR nodes.

\section{Mathematical Simulation}

Simulation Environment And Performance Indicators. This paper refers to an Ad Hoc Network Model and achieves its results using OPNET software. We set the parameters of our simulation to:

Our network covers an area of $3 * 3 \mathrm{~km}^{2}$ with 30 nodes and a distance of communication between two nodes of $300 \mathrm{~m}$. We use the MAC layer with an IEEE802.11 protocol, implementing CSMA/CA mode access. The physical layer uses a spread spectrum and frequency hopping. The network layer uses a table-driven routing protocol OLSR.

Our experiment uses two models of running nodes. Firstly, the nodes run without a regular model with its speeds having random values. We test all of this before adapting the data. Secondly, the nodes use an adaptive model to adjust the rate of the transmitting packets in order to test the improved algorithm. We can evaluate the network performance in two ways:

The average success rate of data transmission, which is defined as OTS (e.g., the rate between the total number of received packets and the total number of transmitted packets). This value reflects the congestion of the error condition for the network. It is an important parameter for measuring the quality of the network, as follows:

$$
\text { OTS }=\frac{\sum_{t} N_{\text {um }} \text { Received }(t)_{t} N_{\text {Send }(t)}}{\sum_{\text {S }}}
$$

The Success Rate of Data Transmission. To verify the success rate of the transmitting packets, our simulations can show different contracting strengths in the design, linearly increasing the number of packets in the network incrementally. Simultaneously, to generate the congestion, transmitting packets follow diagonal paths. As before, we iterate our experiment 20 times in order to take an average. Fig. 8 shows our results. It can be seen that as we advance the simulation, the contracting strength increases and the success rate of the data transmission rapidly declines in the network. After improving and adopting an adaptive contracting strength, the success rate of transmission declines slower and improves. 


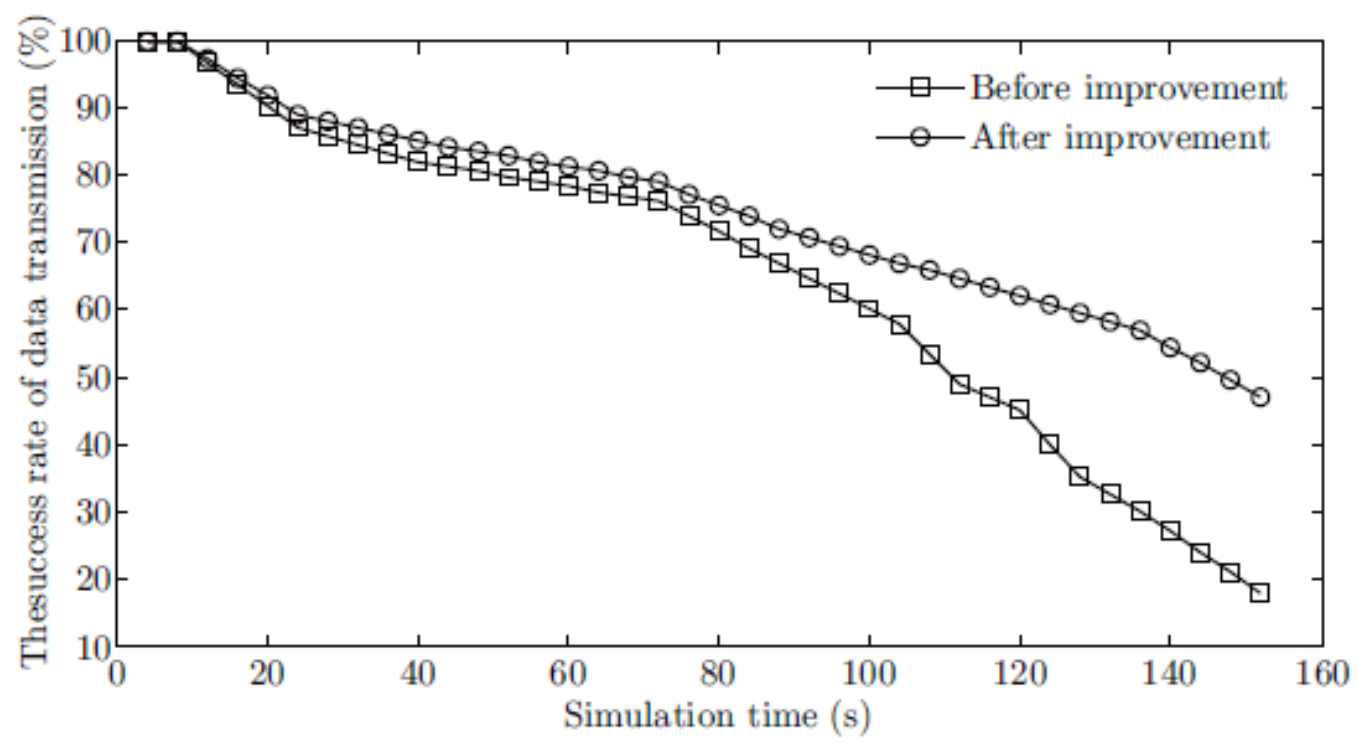

Fig.8 The success rate of data transmission

\section{The Network Throughput}

Network throughput is a vital test for network performance. Using our data, we can see the comprehensive performance of the network and its packet loss rate, the maximum capacity of the network and so on. Fig.9 shows our results. As we can see from Fig.9, when our simulation advances, the contracting strength increases. The extremum of the network throughput is no longer the same as before, varying by a factor of about one-quarter.

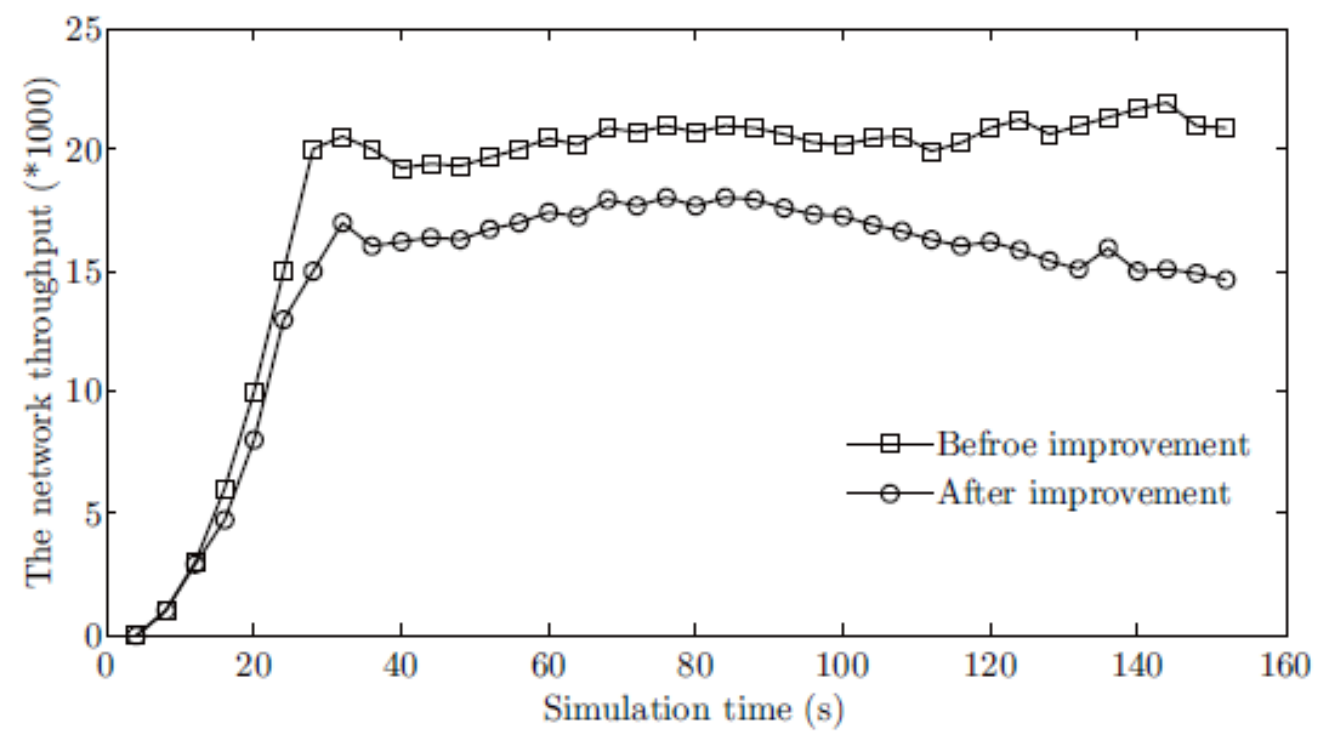

Fig.9 The network throughput

\section{Conclusion}

This paper uses the perspective of mathematical set to get the MPR set, makes the one-hop neighbor nodes to connect into a collection subset and calculates remaining independent subset to get the MPR sets. So we can get the least number of MPR set. This method is more suitable for the network whose nodes move faster and the node density is relatively large. The experiments show that the algorithm can improve the success rate of the data transmission and enhance the network throughput. 


\section{Acknowledgement}

The corresponding author of this paper is Zhang Hong. This paper is supported by the Natural Science Foundation of China (Project No. 61503411).

\section{References}

[1] Haitao Zhao, Hongbo Zhu, Nanjie Liu, Research on capacity-aware routing optimization for wire-less multimedia mesh network [J], Chinese Journal of Scientic Instrument, 34(6), 2013, 1201-1208

[2] Hongyan Qian, Bing Chen, Jiabin Yuan, Enhanced routing protocol on AODV with load alance and delay restriction [J], Journal of Nanjing University of Science and Technology, 37(1), 2013,25-31

[3] Tuanfa Qin, Suyun Liao, Huiping Luo, A network coding-aware routing protocol in wireless mesh network [J], Journal of Beijing University of Posts and Telecommunications, 32(1), 2009, 14-18

[4] Shamsi M, Saad P B, Ibrahim S B, et al. Fast Algorithm for Iris Localization Using Daugman Circular Integro Differential Operator[C] //Proc of the 2009 International Conference on Soft Computing and Pattern Recognition. Washington, DC: IEEE Computer Society, 2009: 393-398

[5] Zhi Sun, Multi-constrained QoS routing protocol based on clustering for wireless mesh network[J], Journal of Computer Applications, 31(3), 2011, 625-628

[6] L. Bononi, M. D. Felice, A. Molinaro et al., Enhancing multi-hop communication over multi-radio multi-channel wireless mesh networks: A cross-layer approach [J], Computer Communications, 34,2011, 1559-1569

[7] Naishuo Tian, Xiuli Xu, Zhanyou Ma, Discrete-time Queuing Theory [M], Beijing: Science Press, 2008 